\title{
Advanced Computational Methods in Molecular Medicine
}

\author{
Alejandro Giorgetti, ${ }^{1,2}$ Paolo Ruggerone, ${ }^{3}$ Sergio Pantano, ${ }^{4}$ and Paolo Carloni ${ }^{5}$ \\ ${ }^{1}$ Department of Biotechnology, University of Verona, Strada Le Grazie 15, 37134 Verona, Italy \\ ${ }^{2}$ Computational Biophysics, German Research School for Simulation Sciences, 52425 Jülich, Germany \\ ${ }^{3}$ CNR-IOM, Unità SLACS and Department of Physics, University of Cagliari, S.P. Monserrato-Sestu km 0.700, \\ 09042 Monserrato, Italy \\ ${ }^{4}$ Group of Biomolecular Simulations, Institut Pasteur de Montevideo, Mataojo 2020, 11400 Montevideo, Uruguay \\ ${ }^{5}$ Computational Biophysics, German Research School for Simulation Sciences, and Institute for Advanced Simulation, \\ Forschungszentrum Jülich, 52425 Jülich, Germany
}

Correspondence should be addressed to Paolo Carloni, p.carloni@grs-sim.de

Received 26 January 2012; Accepted 26 January 2012

Copyright (C) 2012 Alejandro Giorgetti et al. This is an open access article distributed under the Creative Commons Attribution License, which permits unrestricted use, distribution, and reproduction in any medium, provided the original work is properly cited.

The dauntingly complex functioning of human cells is often the outcome of several molecular processes. Understanding such processes is crucial for modern drug discovery, defining interaction cascades, assessing the effects of mutations changes in local concentrations of ligands, and so forth. Computational methods, from systems biology to bioinformatics and molecular simulation, allow to access features difficult or impossible to be measured. Models (if properly validated against experimental data) help understand the intricate molecular mechanisms of life processes. Bolstering the predictive power of these models calls upon the computational biologist to improve algorithms and methods. This issue reports on procedures and on applications facing current challenges in computational biology.

Modern biological sciences are becoming more and more multidisciplinary. At the same time, theoretical and computational approaches gain in reliability and their field of application widens. O. Fisette at al. discuss recent advances in the areas of solution nuclear magnetic resonance (NMR) spectroscopy and molecular dynamics (MD) simulations that were made possible by the synergistic combination of both methods.

Interaction of proteins is of vital importance for many cellular processes and when altered may cause significant health problems, thus the availability of reliable tools to predict and study the determinants of protein-protein interactions is needed. In this regard, X. -Y. Meng et al. present a newly adapted, computationally efficient Brownian Dynamics- (BD-) based protein docking method for predicting native protein complexes. The approach includes global
BD conformational sampling, compact complex selection, and local energy minimization. A shell-based grid force field represents the receptor protein and solvation effects, partially considering protein flexibility.

Hybrid quantum mechanics/molecular mechanics (QM/ $\mathrm{MM}$ ) calculations are routinely used to study quantum mechanical processes in biological systems. J. Kang et al. present a review paper describing an UNIX shell-based interface program connecting two widely used QM and MM calculation engines, GAMESS and AMBER. The tool was used to investigate a metalloenzyme, azurin, and PU.1-DNA complex and mechanisms of hydrolysis (editing reaction) in leucyl-tRNA synthetase complexed with the mis-aminoacylated tRNALeu. The authors investigate the influence of environmental effects on the electronic structure.

Electron transfer in proteins constitutes key steps in several biological processes, ranging from photosynthesis to aerobic respiration. T. Hayashi and A. Stuchebrukhov investigate electron tunneling in NADH : ubiquinone oxidoreductase (Complex I), a key enzyme in cellular respiration as an entry point of the electron transport chain of mitochondria and bacteria, by evaluating the transition flux between donor and acceptor at atomistic resolution. The study suggests that the diffusion of internal water molecules dynamically controls tunneling efficiency.

Methionine sulfoxide reductases (Msr), if reduced by their biological partner, thioredoxin (Trx), act extremely efficient as members of the cellular antioxidant defense system. To understand the Trx-induced reduction mechanism, structural aspects of the intermolecular complex 
formation between mammalian MsrB1 and Trx were studied by Dobrovolska et al., combining NMR spectroscopy and biocomputing. According to the authors, the intermediate MsrB1/Trx complex is stabilized by interprotein $\beta$-layer. The complex formation accompanied by distortion of disulfide bond within MsrB1 facilitates the reduction of oxidized MsrB1.

Integrins have been shown to play a crucial role in promoting tumor growth and metastasis, regulating angiogenesis - the growth of new blood vessels - and lymphangiogenesis - the growth of new lymphatic vessels. Thus, the work of $\mathrm{M}$. Freindorf and coworkers on the binding of a series of hormone analogues to the $\alpha \mathrm{v} \beta 3$ integrin represents a further contribution to the understanding of such fundamental regulators of biological tissues. Using QM/MM methods the authors identified for the ligands considered here multiple modes of binding near the arginine-glycine-aspartate (RGD) recognition site of $\alpha \mathrm{v} \beta 3$ integrin. This observation indicates that there may be subtle changes in the integrin-binding site that can govern binding to alternate sites, influencing downstream signaling pathway.

The paper by F. Fogolari et al. discusses technical aspects of molecular dynamics simulations in the aqueous phase performed at high solute concentrations. These conditions are often present in molecular recognition processes. The paper provides specific examples, ranging from the association of beta- 2 microglobulins to the binding of hydrogen peroxide to its target glutathione peroxidase.

Membrane proteins are the principal targets of the available drugs in the market, yet structural information is available for very few of them. Feature predictions are therefore very important for drug development. D. Mukherjee et al. use Artificial Neural Network (ANN) for the prediction of mechanisms of intracellular protein sorting.

Martinez-Archundia et al. uncovered the role of key residues for the function of a membrane receptor, that is, the human M3 muscarinic acetylcholine receptor. The threedimensional structure of the receptor was built by homology modeling, using the crystallographic structure of bovine rhodopsin as a template. Site mutagenesis experiments were performed to assess the role of two residues, F222 and T235, which are strong candidates for the recognition of ligands. The combined experimental-computational approach confirmed the involvement of both residues in modulating the binding affinity of the ligand.

A rapidly growing number of protein structures is currently deposited in the PDB database in the ambit of structural genomics consortiums. However, the molecular function of the proteins may be neither known nor validated by experiment. Schüller and coworkers apply an integrated bioinformatics tool to transcriptional regulators controlling the expression of genes involved in diverse biological functions of bacteria. They provide a functional classification to factors with known 3D structure but unknown function.

In closing this survey, we would like to mention that back in the late forties, Linus Pauling and coworkers published a paper in Science on "sickle cell anemia, a molecular disease." This contribution kicked off the field of experimental molecular medicine. More than sixty years after Pauling's seminal paper, we observe that computational approaches have become extremely powerful tools, complementary to experiment, to uncover molecular mechanisms of diseases and to thrive strategies to counteract them.

\section{Acknowledgment}

German Research School for Simulation Sciences is a joint venture of RWTH Aachen University and Forschungszentrum Jülich, Germany.
Alejandro Giorgetti

Paolo Ruggerone Sergio Pantano Paolo Carloni 

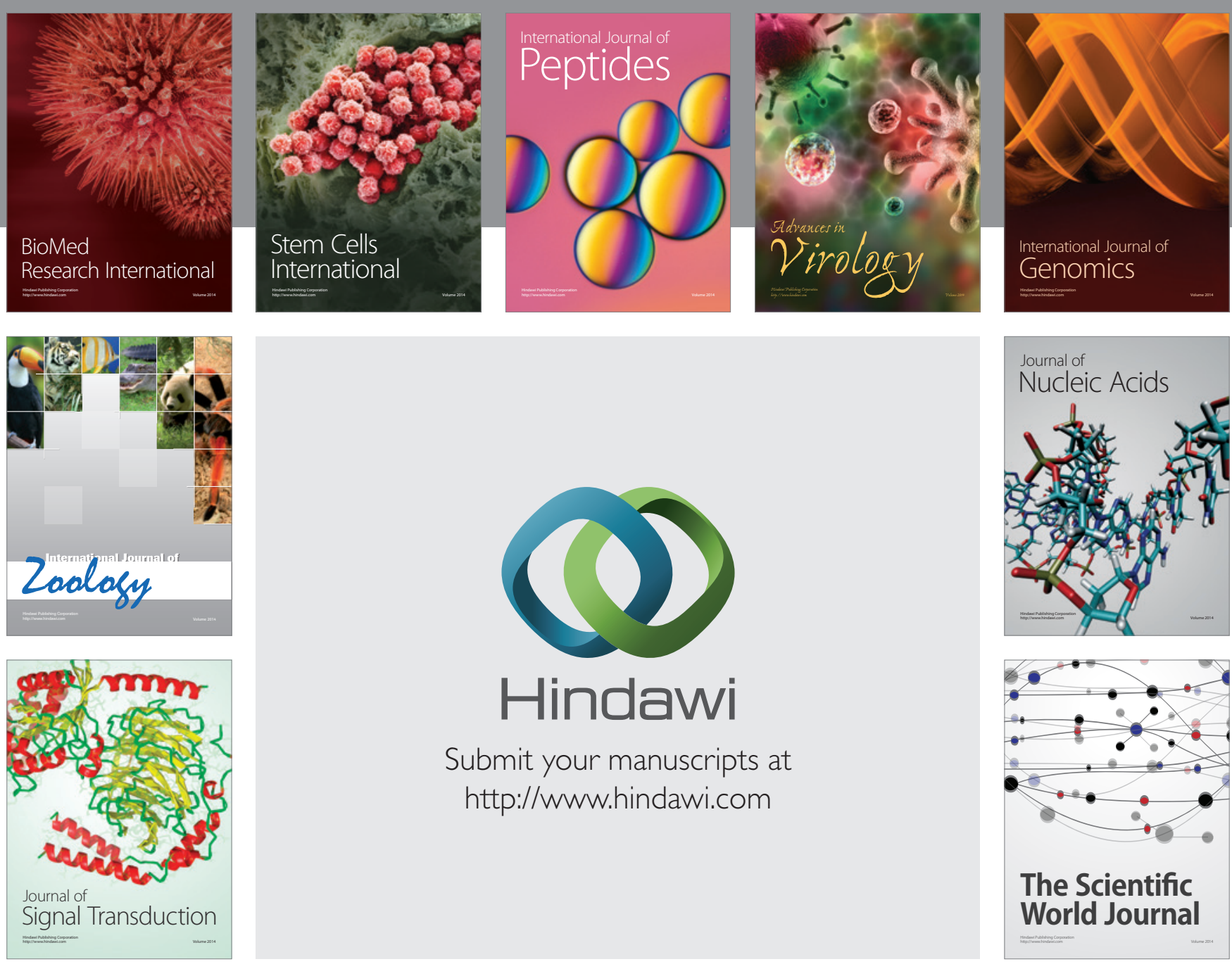

Submit your manuscripts at

http://www.hindawi.com
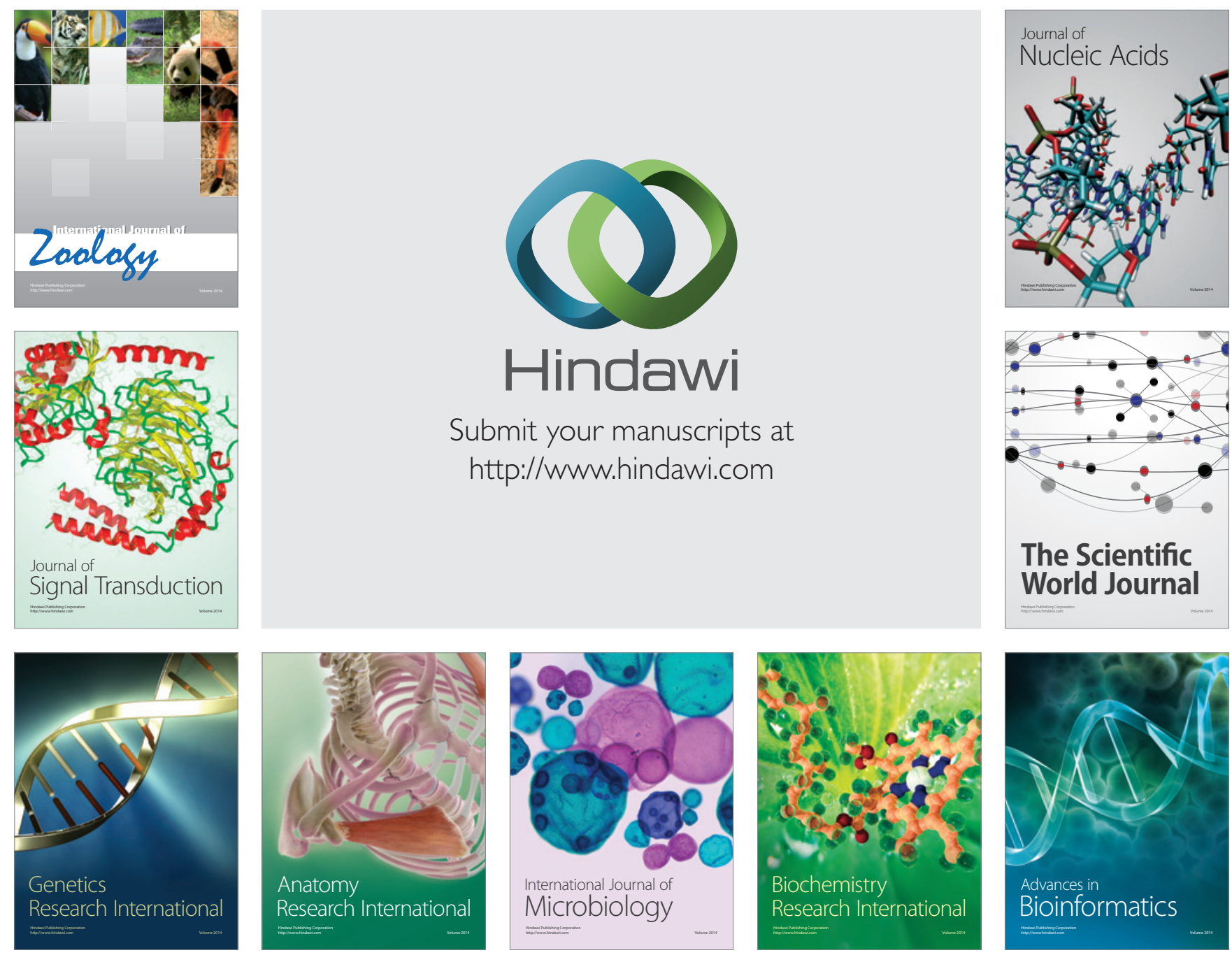

The Scientific World Journal
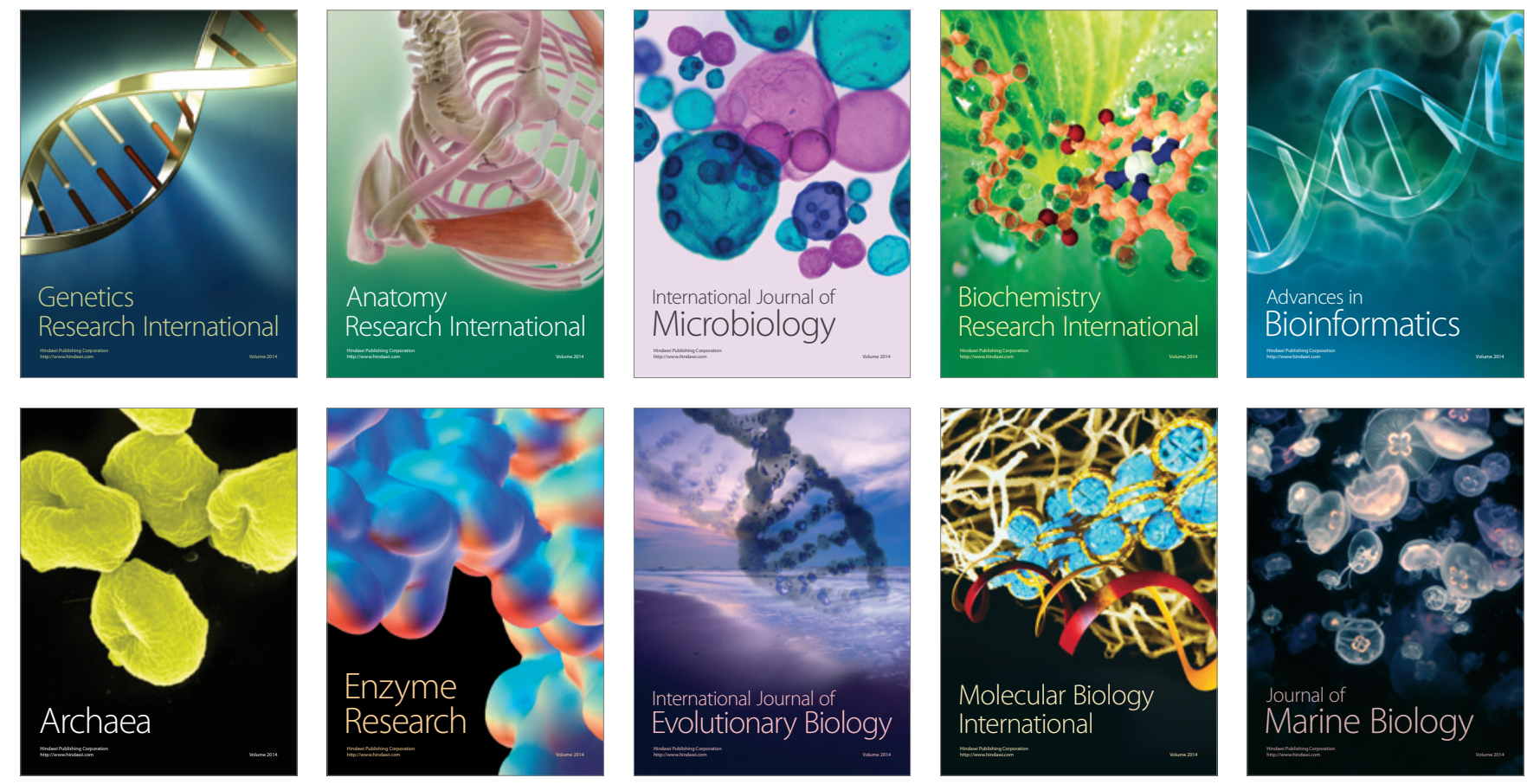